\title{
A Pictorial Review on Reversible Splenial Lesions
}

\author{
Arpita Sahu ${ }^{2}$ Shilpa Sankhe ${ }^{1} \quad$ Kartik Mittal $^{1} \quad$ Namita Kamath $^{1} \quad$ Nandakumar PG $^{2}$ Amit Sahu ${ }^{1}$ \\ ${ }^{1}$ Department of Radiodiagnosis and Imaging, King Edward \\ Memorial Hospital and Seth GS Medical College, Mumbai, \\ Maharashtra, India \\ 2Department of Radiodiagnosis, Tata Memorial Hospital, Homi

\begin{abstract}
Address for correspondence Arpita Sahu, MD, Department of Radiodiagnosis, Tata Memorial Hospital, B-308, Aradhana CHS Ltd, G D Ambekar Marg, Dadar East, Mumbai - 400 014, Maharashtra, India (e-mail: drarpitasahu@gmail.com).
\end{abstract}

Bhabha National Institute, Mumbai, Maharashtra, India

Indian J Radiol Imaging 2021;31:3-9.

\begin{abstract}
Keywords

- diffusion restriction

- reversible

- splenium

Splenium of corpus callosum can be involved in a variety of pathologies causing reversible or irreversible damage. Magnetic resonance imaging (MRI) is a useful investigation to evaluate the same. In spite of the differing etiologies implicated, MRI findings can be quite common. We review the reversible causes of diffusion restriction involving the splenium of corpus callosum and highlight the etiopathologic mechanisms implicated in these pathologies. We further discuss these pathologies in entirety with relevant clinical and laboratory findings helping make definitive diagnosis and guiding appropriate management.
\end{abstract}

\section{Introduction}

The corpus callosum is a very thick bundle of nerve fibers with more than 300 million compact white matter fibers containing both myelinated and unmyelinated axons. ${ }^{1,2}$

It comprises four parts:

1. the reflected anterior portion, or the rostrum;

2. the genu, or the anterior bulbar end;

3. the splenium, or the posterior rounded end; and

4. the body, which lies between the genu and the splenium. ${ }^{3}$

Splenium is the posterior part of the corpus callosum. In Greek, this word means a bandage strip tied around an injury.

Splenium denotes the posterior part of the corpus callosum. The word splenium in Greek means a bandage strip tied around an injury. ${ }^{1}$

Many pathologies affect the splenium of corpus callosum that could be categorized as infective (tuberculosis, influenza), metabolic (hypoglycemia, hypernatremia), toxin induced (Marchiafava-Bignami), vascular (cerebral venous thrombosis (CVT), migraine, posterior reversible encephalopathy syndrome, PRES), autoimmune disorder (systemic lupus erythematosus, SLE), trauma (diffuse axonal injury,

published online April 17, 2021
DOI https://doi.org/

$10.1055 / \mathrm{s}-0041-1729127$

ISSN 0971-3026
DAI), medications (phenytoin), and miscellaneous (malnutrition, vitamin B12 deficiency, hemolytic uremic syndrome, HUS, high-altitude cerebral edema and status epilepticus).

\section{Classification of Etiology}

I. Infective:

- Tuberculosis

- Influenza

II. Metabolic:

- Hypoglycemia

- Hypernatremia

III. Toxic:

- Marchiafava-Bignami syndrome

IV. Vascular:

- PRES

- CVT

- Migraine

v. Autoimmune:

- SLE

VI. Trauma:

- DAI

VII. Medications:

- Phenytoin (c) 2021. Indian Radiological Association

This is an open access article published by Thieme under the terms of the Creative Commons Attribution-NonDerivative-NonCommercial-License, permitting copying and reproduction so long as the original work is given appropriate credit. Contents may not be used for commercial purposes, or adapted, remixed, transformed or built upon. (https://creativecommons.org/licenses/by-nc-nd/4.0/).

Thieme Medical and Scientific Publishers Private Ltd. A-12, Second Floor, Sector -2, NOIDA -201301, India 
VIII. Miscellaneous:

- Status epilepticus

- Malnutrition

- High-altitude cerebral edema

Our aim is to discuss various pathologies causing diffusion restriction in the splenium that shows reversibility during the course of treatment. The vulnerability of splenium to excitotoxic injury is due to the large number of glutamate receptors and high enzymatic activity making it prone to cytotoxic edema and hence diffusion restriction. This excitotoxic edema is thought to be intramyelinic and is not neurotoxic and thus reversible in most of the cases.

The excess glutamate in case of excitotoxic injury comes from one of the three possible ways:

1. Either decreased uptake at the synapse

2. Increased release because of excessive depolarization of neuronal cell membrane

3. Leak from the axonal membrane disruption. ${ }^{4}$

\section{Tuberculous Meningitis}

Tuberculosis is one of the most common infectious diseases in developing countries. Disseminated tuberculosis may affect the central nervous system and lead to tuberculous meningitis (TBM). Patient generally presents with fever, altered sensorium, coma, and stroke like episodes.

Ischemic stroke is commonly seen with TBM but rarely it results in hemorrhagic infarcts. It is attributed to both arterial and venous thrombosis. Stroke in TBM occurs in 15 to $57 \%$ of patients. It is regarded as a poor prognostic predictor of TBM.

Magnetic resonance imaging (MRI) can show tuberculomas, leptomeningeal, and basal cisternal enhancement, hydrocephalus, and infarcts (-Fig. 1).

Although the corpus callosum is involved in various pathologies, it is relatively immune to ischemic injury. One accepted explanation is due to its rich blood supply from both the anterior and posterior circulations. In a study by Chrysikopoulos et al, the authors described the splenium as being most commonly involved in thromboembolic causes of callosal infarction, very likely due to the predilection for posterior cerebral artery versus anterior cerebral artery territory ischemia. $^{5}$

The pathophysiological mechanisms implicated in cerebral vasculitis are multiple and can include a direct action of the infectious agent on the vessel wall, blood-brain barrier damage by the cytokines, immunological involvement by inducing antigen expression on endothelial cells and forming immune complexes. In late stage, organization of basal exudates may strangulate the vessels leading to vascular narrowing and focal weakness. Vascular involvement starts in adventitia and progressively encroaches to involve the entire vessel wall constituting panarteritis tuberculosa. The uncommon complications include aneurysmal dilatation, mycotic aneurysm, thrombus formation, and venous sinus thrombosis.
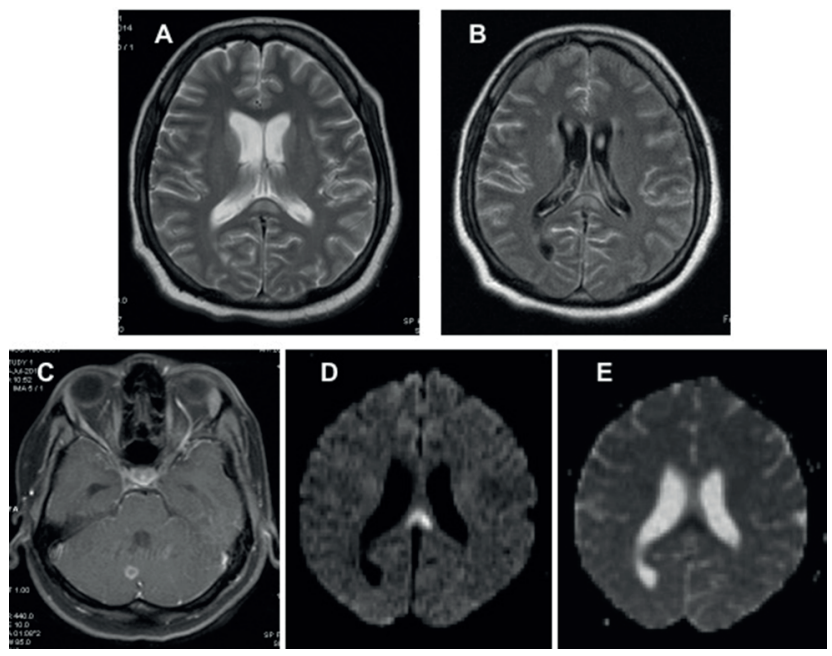

Fig. 1 A 41-year-old male with pulmonary Koch's with meningitis. Magnetic resonance imaging shows $(\mathbf{A}-\mathbf{C})$ an area of T2 hyperintensity with diffusion restriction in the splenium of corpus callosum. This suggests a vasculitic infarct due to tubercular meningitis. (D) Hyperintense signal in splenium of corpus callosum and leptomeningeal enhancement on fluid attenuated inversion recovery postcontrast. (E) A ring enhancing tuberculoma in the right cerebellar hemisphere.

The pathophysiological mechanisms involved in cerebral vasculitis are multiple and are as follows: a direct effect of the organism on the vessel wall, blood-brain barrier damage by the cytokines, antigen expression on endothelial cells and forming immune complexes by various immune-mediated reactions.

At later stage, basal exudates organized and obliterate the vessel leading to luminal narrowing. The adventitia is involved at first and progressively involves the entire vessel wall resulting in panarteritis tuberculosa.

Few rare complications include aneurysmal dilatation of vessel, mycotic aneurysm formation, and cerebral venous sinus thrombosis. ${ }^{6,7}$

\section{Influenza}

Influenza is one of the most common respiratory tract infections that may lead to epidemics especially in winter season and cause flu-like symptoms. Disease spectrum includes Reye syndrome, hemorrhagic/necrotizing encephalopathy, and influenza-associated encephalitis.

Multiple areas of T2 hyperintensity and restricted diffusion are seen in the spectrum of influenza-associated encephalitis/encephalopathy involving subcortical white matter, cortex, and the splenium of the corpus callosum. ${ }^{8}$ Similarly, symmetrical restricted areas may be seen in the basal ganglia, thalamus, and brainstem, cerebellum in acute necrotizing encephalopathy ${ }^{9}$ (-Fig. 2)

Splenial lesion is commonly seen in influenza and probably is a result of intramyelinic edema and inflammatory infiltrates. Intramyelinic edema results in separation of myelin layers leading to reversible diffusion restriction. ${ }^{10}$ 


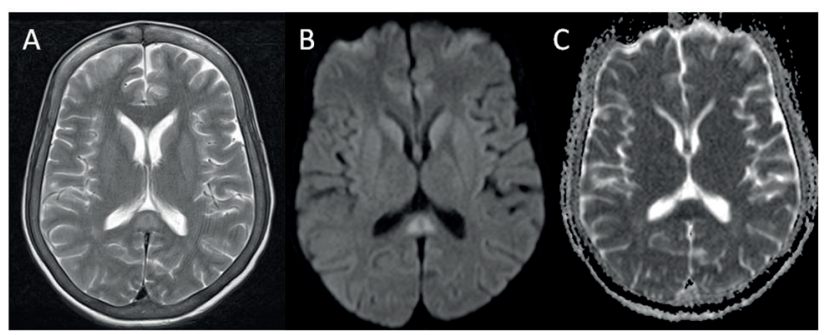

Fig. 2 A 34-year-old male patient with fever and neck rigidity, diagnosed to have influenza; T2-weighted axial magnetic resonance imaging shows (A) hyperintensity in the splenium of corpus callosum with diffusion restriction in the corresponding diffusion-weighted imaging, apparent diffusion coefficient maps (B, C).
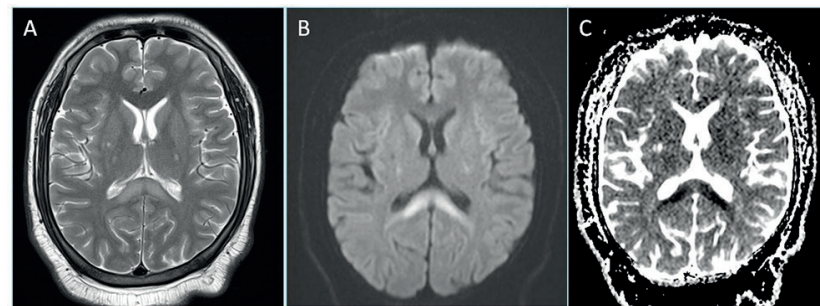

Fig. 3 A 33-year-old female patient presented with loss of consciousness and blood sugar of $38 \mathrm{mg} / \mathrm{dL}$ (hypoglycemia). T2-weighted axial magnetic resonance imaging shows $(\mathbf{A})$ hyperintensity in the splenium of corpus callosum with $(\mathbf{B}, \mathbf{C})$ diffusion restriction in the corresponding diffusion-weighted imaging and apparent diffusion coefficient maps.

\section{Hypoglycemia}

Hypoglycemia is defined as an abnormally low plasma glucose concentration that leads to harmful effects. The plasma glucose cutoff level is less than $70 \mathrm{mg} / \mathrm{dL}$ (normal range: $70-110 \mathrm{mg} / \mathrm{dL}$ ). Most common reason for hypoglycemia in this diabetic era is iatrogenic hypoglycemia, which is associated with insulin and oral hypoglycemic agents. ${ }^{11}$

Glucose serves as the sole metabolic fuel for central nervous system, especially the gray matter where it is the only source of energy. Any reduction in the metabolic demand will result in symptoms ranging from palpitations, sweating, and dizziness to confusion, altered sensorium, focal deficits, seizures, and encephalopathy in severe situations. ${ }^{11}$

The commonly affected areas are hippocampus, occipital and parietal cortex, pons, basal ganglia, corpus callosum, and internal capsule. On MRI, these areas show T1 hypointensity, T2/fluid attenuated inversion recovery (FLAIR) hyperintensity, and restricted diffusion on diffusion-weighted imaging (DWI) if cytotoxic edema has become evident $t^{12,13}$ ( - Fig. 3 ).

Reduction in brain plasma glucose level will affect the cellular fluid mechanisms and cause intramyelinic edema. Subsequent energy depletion will lead to intracellular alkalosis, arrest of protein synthesis and at last lead to membrane ion pump failure, thus converting vasogenic edema to cytotoxic edema. ${ }^{13}$
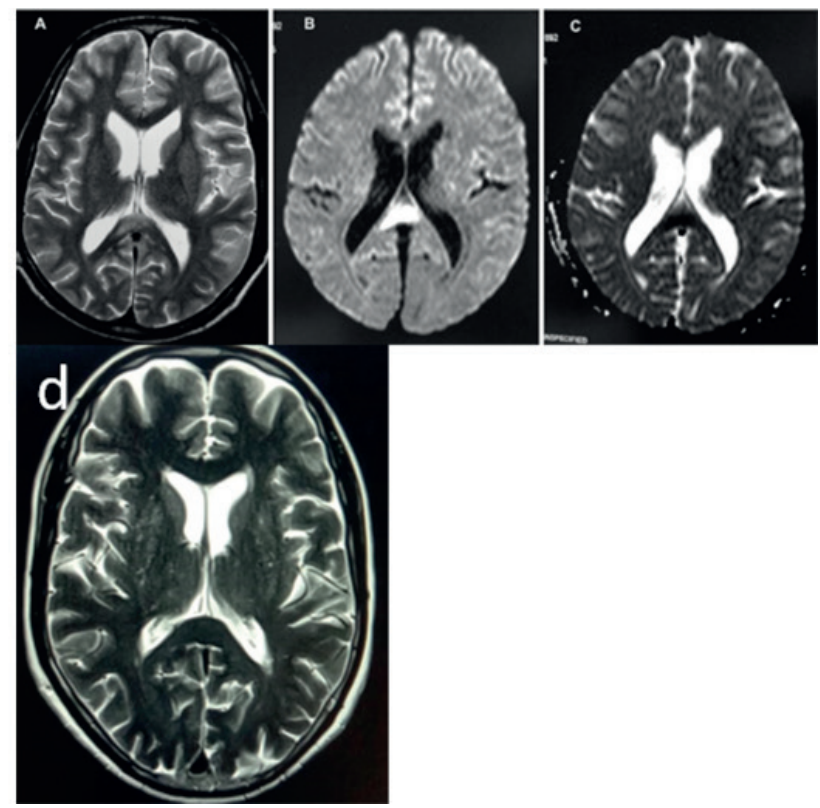

Fig. 4 A 26-year-old male patient with recent history of alcohol binge; T2-weighted axial magnetic resonance imaging shows (A) hyperintensity in the anterior and middle fibers of splenium of corpus callosum with diffusion restriction in the corresponding diffusion-weighted imaging and apparent diffusion coefficient maps (B, C). (D) T2-weighted image of the same patient after 3 months shows complete resolution of the splenial hyperintense lesion.

\section{Marchiafava-Bignami Disease}

Marchiafava-Bignami disease (MBD) is a rare form of toxic demyelination of the corpus callosum associated with chronic alcoholic consumption, although nutritional deficiencies have also been thought as a cause of this disease entity. It was first described by Marchiafava and Bignami in 1903. Acute MBD patients may present with generalized tonic-clonic seizures, altered sensorium, and coma. It involves not only the corpus callosum but also the hemispheric white matter.

In acute MBD, there is classical involvement of the central portion of the splenium of the corpus callosum with sparing of the dorsal and ventral layers in the form of $\mathrm{T} 2$ hyperintensity and diffusion restriction. On follow-up, there can be normal signal intensity of the involved region or development of cystic lesion that is suggestive of chronic changes. This diffusion restriction is caused by edema and early myelin damage that if remyelinates completely is suggestive of reversibility of the lesion (-Fig. 4). ${ }^{14,15}$

\section{Posterior Reversible Encephalopathy Syndrome}

PRES is a neurotoxic condition that occurs in eclampsia, transplant patients on cyclosporine, autoimmune disease such as SLE or Wegener's granulomatosis, HUS, thrombotic thrombocytopenic, and postchemotherapy. Patients initially present with headache along with altered vision, altered consciousness, and convulsion. 
MRI shows T2 hyperintensity in the parietal and occipital cortex and white matter. It can also involve the frontal, parietal, and occipital lobes, with lesser involvement of the temporal lobes. Less commonly seen pattern is vasogenic edema in the watershed or anastomotic border zone (-Fig. 5). ${ }^{16}$

Cerebral autoregulatory mechanisms work using both myogenic and neurogenic components for constant brain perfusion. In PRES, there is poor myogenic response due to passive over distention of the vessel due to high blood pressure or toxic effects on the endothelium. The posterior circulation is most vulnerable as they are poorly innervated by the sympathetic system and finally there is leakage of fluid into the interstitium and resultant vasogenic edema. ${ }^{17}$

Severe vasogenic edema may progress to cytotoxic edema. DWI hyperintensity is seen in PRES with either low, normal, or elevated apparent diffusion coefficient (ADC) values; such variable ADC values are attributed to a phenomenon called pseudonormalization that results from intravoxel averaging of both cytotoxic and vasogenic edema in areas affected by PRES. Restricted diffusion in cytotoxic edema causes decrease in ADC values and vasogenic edema causes elevation of the ADC values, the effects of cytotoxic edema and vasogenic edema cancel each other or one of the effects overrides the other when the two are combined at the subvoxel level. Restricted diffusion in PRES is irreversible when the effects of cytotoxic edema begin to dominate and ischemia or infarct may result from it. ${ }^{17}$

According to Ay et al, diffusion restriction is seen in areas of massive unchecked vasogenic edema due to increased tissue pressure causing abnormality in the microcirculation and there by cytotoxic edema. ${ }^{18}$

\section{Cerebral Venous Thrombosis}

CVT is a condition of focal or diffuse occlusion of venous channels of the brain that includes cortical vein thrombosis and deep cerebral vein thrombosis. CVT, generally a disease of the young, is comparatively rare to arterial stroke. Its clinical evolution is different from other stroke subtypes with symptoms varying from headaches, nausea, vomiting to decreased and
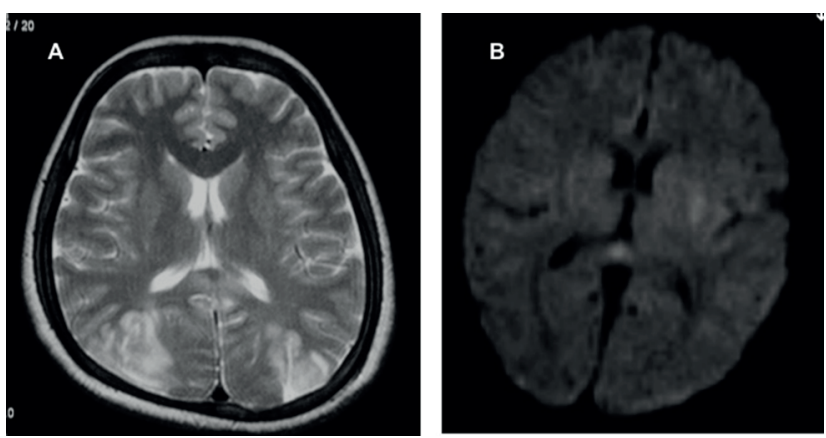

Fig. 5 A 23-year-old postpartum female with a history of preeclampsia now presented with altered sensorium magnetic resonance imaging shows (A) on T2 axial image bilateral symmetrical parieto-occipital hyperintensity involving both gray and white matter with focal hyperintensity seen in the region of the splenium. (B) The lesion in the splenium shows diffusion restriction. Imaging features are in favor of posterior reversible encephalopathy syndrome. altered conscious with signs of papilloedema, cranial nerve palsies, or focal neurological deficits. Pregnancy, malignancy, dehydration, and prothrombotic hematological conditions may predispose to CVT. ${ }^{19}$

Most commonly used method for diagnosis of CVT is time of flight MR venography. In approximately half of all patients increased T2 signal intensity associated with CVT is seen with diffusion weighted images showing restricted diffusion. MRI with GRE sequences is sensitive for parenchymal hemorrhages that are flame-shaped irregular zones of lobar hemorrhage typically with cortical and subcortical extension. ${ }^{20}$ Restricted diffusion in splenium is seen in cases of internal cerebral vein and straight sinus thrombosis (-Fig. 6).

Increase in back pressure results in reversal of flow and increase in velocity in the venous sinuses leading to bloodbrain barrier disruption contributing to cytotoxic edema. Neovascularization due to opening of new channels and recanalization of the occluded veins cause accelerated myelination, leading to reversible splenial lesion. ${ }^{21}$

\section{Systemic Lupus Erythematosus}

SLE is a complex autoimmune connective tissue disease with multisystem involvement ranging from kidney, hematopoietic systems, joints, skin to central as well as peripheral nervous with overall increased female predilection. The disease commonly presents with neurological symptoms in the form of headache, limb weakness, and seizure and sometimes with hemiparesis, cranial nerve, and sensory involvement.

Autoantibodies against host antigens produce inflammatory process and noninflammatory thickness of small vessels with intima proliferation, small vessel obstruction and infarcts, embolism or hemorrhage due to vasculitis. ${ }^{22}$
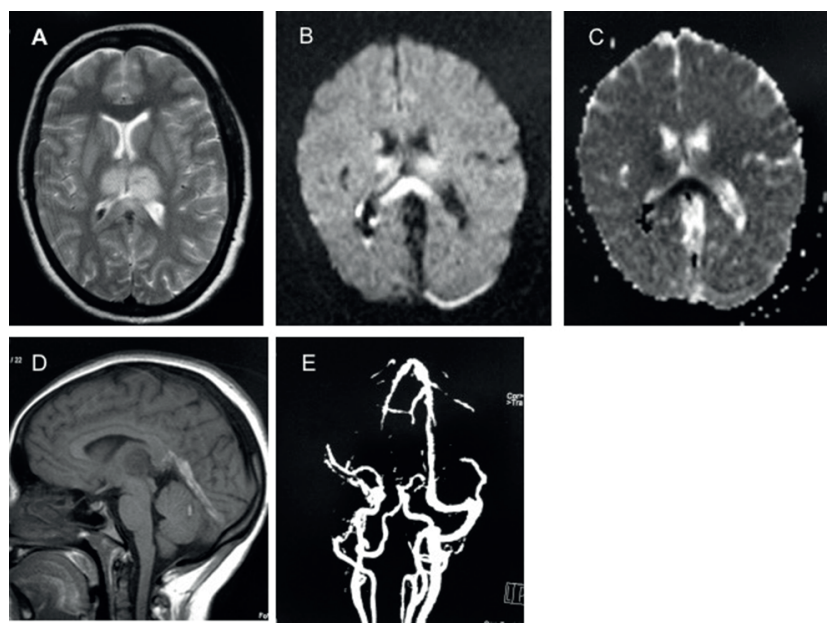

Fig. 6 A 30-year-old female on oral contraceptive pills came with altered sensorium; T2-weighted axial magnetic resonance imaging shows (A) hyperintensity noted in bilateral thalami and splenium of corpus callosum. (B and $\mathbf{C}$ ) Corresponding diffusion-weighted imaging and apparent diffusion coefficient map show restricted diffusion. (D) T1-weighted sagittal image shows loss of flow void in the straight sinus with a hyperintense focus in the right cerebellar hemisphere s/o hemorrhagic infarct. (E) Three-dimensional time of flight venogram confirmed the findings of straight sinus and right transverse sinus thrombosis. 
MRI shows various patterns of disease in the form of white matter hyperintensity that may be small focal area ovoid in shape suggestive of microinfarct, large area in the form of infarct and area of hyperintensity in the cortical gray matter with atrophy of the brain that is disproportionate to the duration of the disease as patients are on steroid ${ }^{23}$ ( - Fig. 7 ).

SLE causes small vessel arteriopathy with reduced flow of blood to the brain and altering the adenosine triphosphate (ATP) pump mechanism. This causes fluid accumulation in the intracellular compartment, leading to diffusion restriction. ${ }^{24}$

\section{Diffuse Axonal Injury}

DAI encompasses a vast majority of intra-axial lesions in traumatic closed head injury from rotational acceleration and deceleration forces producing diffuse shear-strain with significant morbidity and mortality.

T2 hyperintense lesions with diffusion restriction are seen usually at the gray-white junction (grade 1 injury), in the corpus callosum (grade 2 injury) and at the dorsolateral aspect of the upper brain stem (grade 3 injury). Direct contact between the skull and brain parenchyma leads to cortical contusion in the temporal and frontal lobes with small areas of hemorrhage identified by signal blooming on gradient-echo images. Involvement of splenium is not uncommon

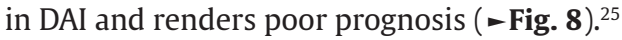

Traumatic brain injury causes parenchymal damage that may be due to the loss of calcium and potassium homeostasis and release of excitotoxic amino acids, free radicals, and tissue acidosis causing neurotoxic edema that may be the cause of extended period of decreased ADC values in traumatic brain injury. ${ }^{26}$

\section{Phenytoin}

Antiepileptic medications are commonly used for seizure disorders. Therapeutic blood level (normal is $10-20 \mathrm{ng} / \mathrm{dL}$ ) monitoring of drug is essential to prevent toxic effects of antiepileptic medications. These patients present with nausea, confusion, nystagmus, gait disturbances, coma, and seizures.

Focal brain lesion in the splenium of the corpus callosum is observed with normal or increased blood levels or sudden
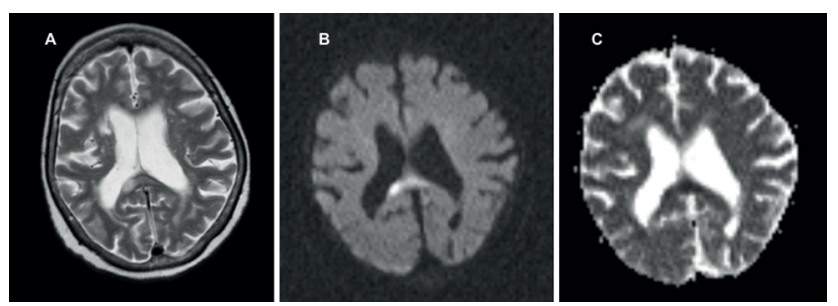

Fig. 7 A 48-year-old female presented with systemic lupus erythematosus. T2-weighted axial magnetic resonance imaging reveals $(\mathbf{A})$ bilateral periventricular discrete hyperintense foci with areas of diffusion restriction (B, C) seen in splenium suggestive of vasculitic infarct and other regions of old sequelae to vasculitis. There is generalized cerebral atrophy.

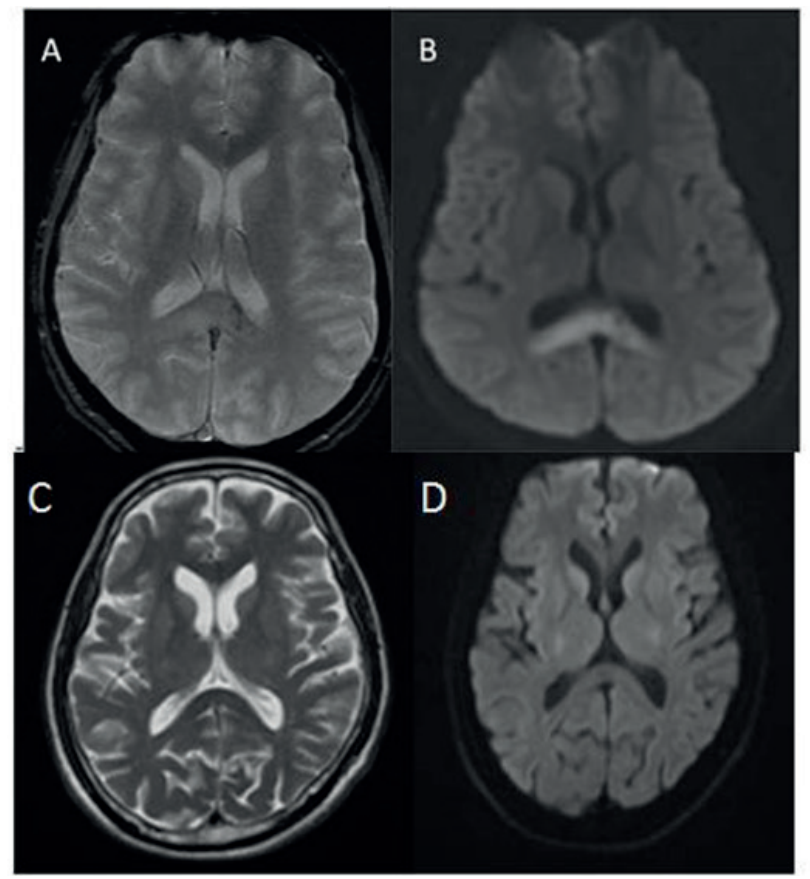

Fig. 8 (A, B) T2 axial image shows hyperintensity with diffusion restriction (B) in the splenium and a hypointense foci in the left half. This represents hemorrhagic foci in a case of diffuse axonal injury. (C, D) T2 and diffusion-weighted imaging follow-up images of the same patient after 9 months show reversibility of the hyperintensity and the restricted diffusion in the splenium.
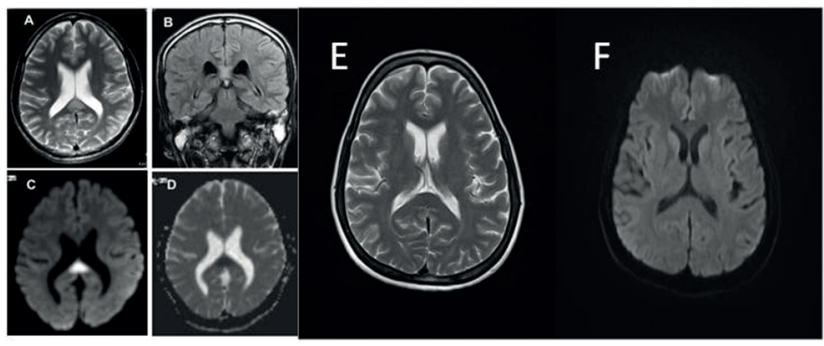

Fig. 9 Known case of chronic seizures on eptoin with blood eptoin level of $50 \mu \mathrm{g} / \mathrm{mL}$. T2-weighted axial magnetic resonance imaging (A) and (B) fluid attenuated inversion recovery coronal image show hyperintensity in the central region of the splenium corresponding diffusion restriction (C, D). Follow-up images (E, F) of T2 and diffusion-weighted imaging of the same patient after 1 year show reversibility of the hyperintensity and the restricted diffusion in the splenium.

withdrawal of antiepileptic medications, such as phenytoin, vigabatrin, and carbamazepine (- Fig. 9).

Seizure activity effects of medications act on the argininevasopressin system and glutamate receptors, leading to excitotoxic injury and reversible cytotoxic edema in astrocytes or myelin sheaths in the splenium, and propagate to the contralateral hemisphere. ${ }^{4}$

Another mechanism suggested is by interfering the intestinal absorption of dietary folate, causing folate deficiency leading to encephalopathy, cerebellar atrophy, myelopathy, and peripheral neuropathy. ${ }^{27}$ 


\section{Epilepsy}

Epilepsy is a disorder that causes multiple and recurrent seizures due to recurrent episodes of paroxysmal brain dysfunction from a sudden, disorderly, and excessive neuronal discharge resulting in transient episodes of sensory or motor neurological dysfunction or psychic dysfunction.

There is a transient increase in signal intensity with swelling on peri-ictal T2-weighted and diffusion-weighted images involving the cortical gray matter and subcortical white matter specifically bilateral cingulate gyri, parieto-occipital, parahippocampal, hippocampus region. Hippocampal atrophy after recurrent seizures has been seen in many cases.

There has been a transient T2/FLAIR hyperintensity with gyral swelling and restricted diffusion, specifically the bilateral cingulate gyri, parahippocampal, hippocampal, and parieto-occipital region. Follow-up MRI with recurrent seizures had shown hippocampal atrophy ( Fig. 10). ${ }^{28}$

Reversible splenial lesions showing diffusion restriction are not uncommon in generalized tonic-clonic seizures. The cause for diffusion restriction is repeated excessive activity of commissural projections from temporal structure, leading to transient disturbance of energy metabolism and ionic transport. ${ }^{29}$

\section{Migraine}

Migraine is a very common condition associated with severe unilateral debilitating headaches accompanied with an aura in some cases with nausea, photophobia, vomiting, and rarely presents with acute neurologic deficits, which may pose diagnostic difficulties.

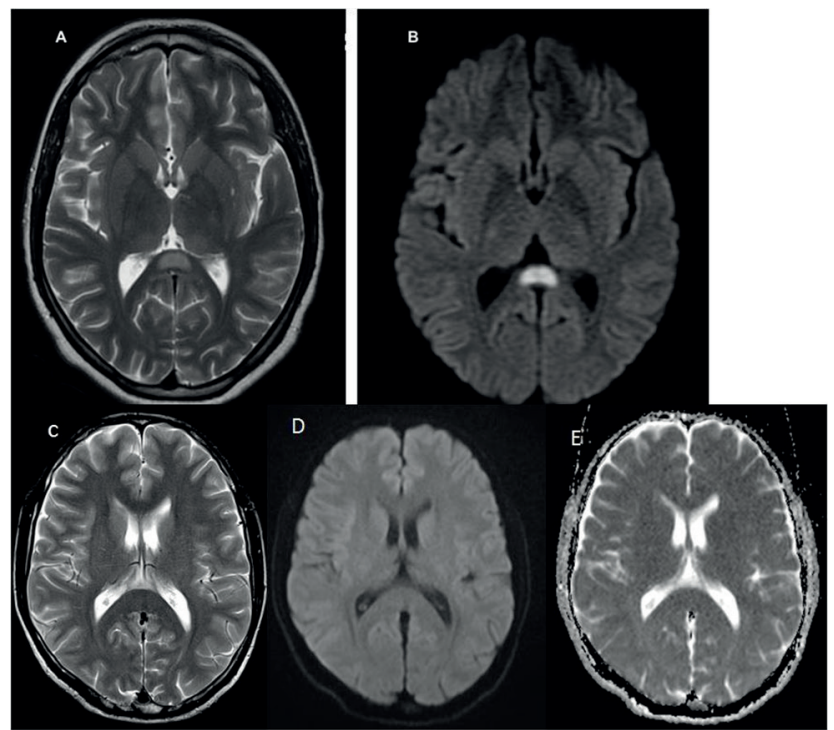

Fig. 10 A 32-year-old female presented with multiple episodes of generalized tonic-clonic seizures. On T2-weighted axial magnetic resonance imaging, $(\mathbf{A})$ shows hyperintensity in the central fibers of the splenium of corpus callosum. (B) Diffusion restriction is seen in the central fibers of the splenium of corpus callosum. (C-E) Image of the same patient after 6 weeks shows complete reversibility of the T2-weighted hyperintensity and restricted diffusion seen in the splenium in the prior study.
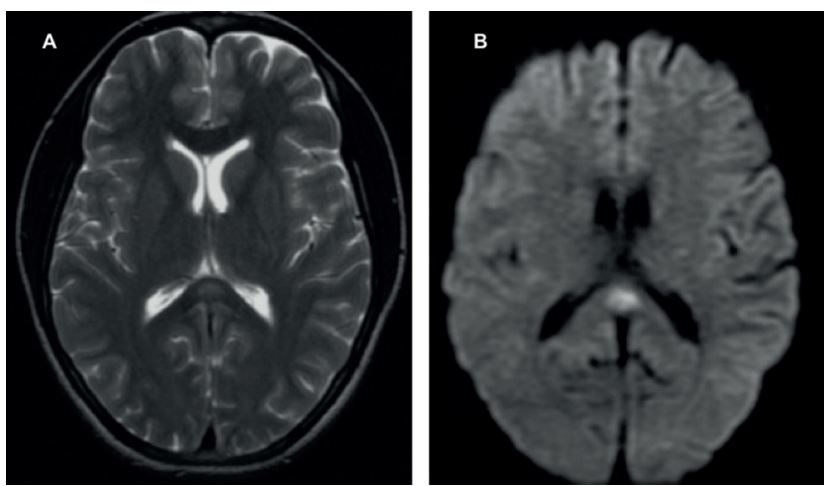

Fig. 11 (A, B) Classical boomerang sign is seen on T2-weighted image and diffusion-weighted imaging. There is hypertrophy of bilateral temporalis muscles (A) finding occasionally seen in migraine.

Many causes have been postulated but changes in cerebral vessel reactivity explain the symptoms with initial vasoconstriction of the cerebral vasculature and cerebral ischemia. This results in T2 hyperintensity in the centrum semiovale similar to small vessel ischemic changes. Later, cerebral vasodilatation results in the pulsatile headache ( - Fig. 11). ${ }^{30}$

These patients also showed a reduction in the gray matter density especially in the bilateral frontal and temporal lobes as compared with healthy controls. ${ }^{31}$ There is a significant increase in risk of silent infarct especially in the posterior circulatory territory of the brain, thus at times affecting the splenium. $^{32}$

Transient vasoconstriction in migraine is more prolonged in posterior circulation including the splenium causing depletion of ATP and poor functioning of $\mathrm{NA}^{+} / \mathrm{K}+$ pump. This causes cellular edema and reduction in the extracellular compartment, leading to transient diffusion restriction. ${ }^{24}$

\section{Conclusion}

Isolated splenial lesion showing diffusion restriction is generally reversible. There are many differentials of reversible splenial lesion and reversibility itself suggests better prognosis. Good clinicoradiological correlation and relevant laboratory investigations help in determining the etiopathogenesis of reversible causes and guide appropriate patient management.

If only single/solitary lesion is seen in splenium, it is almost always reversible and there is no need for further follow-up. Multiple lesions may require follow-up depending on the clinical setting of the individual condition.

\section{Financial Support and Sponsorship \\ Nil.}

\section{Conflict of Interest}

The authors have no conflict of interest.

\section{References}

1 Knyazeva MG. Splenium of corpus callosum: patterns of interhemispheric interaction in children and adults. Neural Plast 2013;2013:639430 
2 Mohammadi MR, Zhand P, Mortazavi Moghadam B, Golalipour MJ. Measurement of the corpus callosum using magnetic resonance imaging in the north of Iran. Iran J Radiol 2011;8(4):218-223

3 Georgy BA, Hesselink JR, Jernigan TL. MR imaging of the corpus callosum. AJR Am J Roentgenol 1993;160(5):949-955

4 Moritani T, Smoker WRK, Sato Y, Numaguchi Y, Westesson PLA. Diffusion-weighted imaging of acute excitotoxic brain injury. AJNR Am J Neuroradiol 2005;26(2):216-228

5 Chrysikopoulos H, Andreou J, Roussakis A, Pappas J. Infarction of the corpus callosum: computed tomography and magnetic resonance imaging. Eur J Radiol 1997;25(1):2-8

6 Boukobza M, Tamer I, Guichard JP, et al. [Tuberculosis of the central nervous system. MRI features and clinical course in 12 cases]. J Neuroradiol 1999;26(3):172-181

7 Oumerzouk J, Hssaini Y, Ait Berri M, Ragabbi A. Fatal tuberculous meningovasculitis. IJSER 2012;3(10):24-27

8 Bulakbasi N, Kocaoglu M, Tayfun C, Ucoz T. Transient splenial lesion of the corpus callosum in clinically mild influenza-associated encephalitis/encephalopathy. AJNR Am J Neuroradiol 2006;27(9):1983-1986

9 Wong AM, Simon EM, Zimmerman RA, Wang HS, Toh $\mathrm{CH}$, $\mathrm{Ng} \mathrm{SH}$. Acute necrotizing encephalopathy of childhood: correlation of MR findings and clinical outcome. AJNR Am J Neuroradiol 2006;27(9):1919-1923

10 Takanashi J, Barkovich AJ, Yamaguchi K, Kohno Y. Influenzaassociated encephalitis/encephalopathy with a reversible lesion in the splenium of the corpus callosum: a case report and literature review. AJNR Am J Neuroradiol 2004;25(5):798-802

11 Kalra S, Mukherjee JJ, Venkataraman S, et al. Hypoglycemia: the neglected complication. Indian J Endocrinol Metab 2013; 17(5):819-834

$12 \mathrm{Kim} \mathrm{JH}$, Choi JY, Koh SB, Lee Y. Reversible splenial abnormality in hypoglycemic encephalopathy. Neuroradiology 2007;49(3):217-222

13 Duberkar D, Lal V. Reversible splenial abnormality in hypoglycemia. Neurol India 2011;59(3):475-476

14 Bano S, Mehra S, Yadav SN, Chaudhary V. Marchiafava-Bignami disease: role of neuroimaging in the diagnosis and management of acute disease. Neurol India 2009;57(5):649-652

15 Ihn YK, Hwang SS, Park YH. Acute Marchiafava-Bignami disease: diffusion-weighted MRI in cortical and callosal involvement. Yonsei Med J 2007;48(2):321-324

16 Bartynski WS, Boardman JF. Distinct imaging patterns and lesion distribution in posterior reversible encephalopathy syndrome. AJNR Am J Neuroradiol 2007;28(7):1320-1327

17 Covarrubias DJ, Luetmer PH, Campeau NG. Posterior reversible encephalopathy syndrome: prognostic utility of quantitative diffusion-weighted MR images. AJNR Am J Neuroradiol 2002;23(6):1038-1048
18 Ay H, Buonanno FS, Schaefer PW, et al. Posterior leukoencephalopathy without severe hypertension: utility of diffusion-weighted MRI. Neurology 1998;51(5):1369-1376

19 Rodallec MH, Krainik A, Feydy A, et al. Cerebral venous thrombosis and multidetector CT angiography: tips and tricks. Radiographics 2006;26(Suppl 1):S5-S18, discussion S42-S43

20 Crawford SC, Digre KB, Palmer CA, Bell DA, Osborn AG. Thrombosis of the deep venous drainage of the brain in adults. Analysis of seven cases with review of the literature. Arch Neurol 1995;52(11):1101-1108

21 Usman U, Wasay M. Mechanism of neuronal injury in cerebral venous thrombosis. J Pak Med Assoc 2006;56(11):509-512

22 Harirchian MH, Saberi H, Najafizadeh SR, Hashemi SA. Evaluation of brain and cervical MRI abnormality rates in patients with systemic lupus erythematosus with or without neurological manifestations. Iran J Radiol 2011;8(3):157-160

23 Aisen AM, Gabrielsen TO, McCune WJ. MR imaging of systemic lupus erythematosus involving the brain. AJR Am J Roentgenol 1985;144(5):1027-1031

24 Moritani T, Shrier DA, Numaguchi Y, Takahashi C, Yano T, Nakai K, Zhong J, Wang HZ, Shibata DK, Naselli SM (2001) Diff usionweighted MR imaging of CNS involvement in systemic lupus erythematosus. Academic Radiology 8:741-753

25 Liu AY, Maldjian JA, Bagley LJ, Sinson GP, Grossman RI. Traumatic brain injury: diffusion-weighted MR imaging findings. AJNR Am J Neuroradiol 1999;20(9):1636-1641

26 Barzó P, Marmarou A, Fatouros P, Hayasaki K, Corwin F. Contribution of vasogenic and cellular edema to traumatic brain swelling measured by diffusion-weighted imaging. J Neurosurg 1997;87(6):900-907

27 Kim SS, Chang KH, Kim ST, et al. Focal lesion in the splenium of the corpus callosum in epileptic patients: antiepileptic drug toxicity? AJNR Am J Neuroradiol 1999;20(1):125-129

28 Kim JA, Chung JI, Yoon PH, et al. Transient MR signal changes in patients with generalized tonicoclonic seizure or status epilepticus: periictal diffusion-weighted imaging. AJNR Am J Neuroradiol 2001;22(6):1149-1160

29 Oster J, Doherty C, Grant PE, Simon M, Cole AJ. Diffusionweighted imaging abnormalities in the splenium after seizures. Epilepsia 2003;44(6):852-854

30 Karaarslan E, Ulus S, Kürtüncü M. Susceptibility-weighted imaging in migraine with aura. AJNR Am J Neuroradiol 2011;32(1):E5-E7

31 Rocca MA, Ceccarelli A, Falini A, et al. Brain gray matter changes in migraine patients with T2-visible lesions: a 3-T MRI study. Stroke 2006;37(7):1765-1770

32 Kruit MC, Launer LJ, Ferrari MD, van Buchem MA. Infarcts in the posterior circulation territory in migraine. The population-based MRI CAMERA study. Brain 2005;128(Pt 9): 2068-2077 